\title{
Computer Optimization of Inhomogeneous Waveguide Transformers
}

\author{
JOHN W. BANDLER, MEMBER, IEEE
}

\begin{abstract}
The problem of designing broadband multisection stepped rectangular waveguide impedance transformers, when the input and output guides have different cutoff frequencies but propagate the same mode, is formulated in general terms for direct optimization by digital computer. The formulation is sufficiently flexible to allow nonideal junction discontinuity effects and mismatched terminations to be taken into account during optimization. Constraints placed on the width, height, or length of any section need be dictated only by considerations for dominant mode propagation and the requirement of small (but not necessarily negligible) junction discontinuities. The objective of the present formulation is a minimax equal-ripple response over a predetermined frequency band satisfying the constraints selected for the particular problem. The ripple search strategy to locate the maximum reflection coefficient within the band and the razor search strategy to minimize it, as described by Bandler and Macdonald [8] in another paper, were employed. Constrained optimum equal-ripple solutions to examples previously published by Young [2]-[4] Matthaei et al. [4] and Riblet [5] are presented. They demonstrate the considerable improvements made possible by the present formulation with regard to performance, reduction in number of sections, and physical size. The approach used in this paper should also find application in the design of broadband microwave matching or equalizing networks consisting of noncommensurate components and for which exact synthesis techniques may be unavailable.
\end{abstract}

\section{INTRODUCTION}

T $\checkmark$ HE PROBLEM of designing broadband multisection stepped rectangular waveguide impedance transformers, when the input and output guides have different cutoff frequencies but propagate the same mode, has already received some attention [1]-[5]. However, the inherent complexity of an exact synthesis of such structures to have broadband performance has led previous authors to formulate approximate design theories based on equal-ripple solutions for homogeneous quarter-wave transformers [6].

Young, who studied one- and two-section transformers [1]-[4], restricted the lengths of the sections to be quarterwave at a common frequency within the band of interest. Riblet [5] specified that each section be quarter-wave at the midband guide wavelength of that section defined by the guide wavelengths at the upper and lower edges of the frequency band. Thus, in general, the sections would be quarterwave at different frequencies. Young's broadband designs resulted from modifications to his exact maximally fiat solutions. Riblet's multisection design procedure involves making

Manuscript received February 4, 1969; revised April 28, 1969. This work was carried out at the University of Manitoba with financial assistance from the Faculty of Graduate Studies of the University of Manitoba and from the National Research Council of Canada.

The author was with the Numerical Applications Group, Department of Electrical Engineering, University of Manitoba, Winnipeg, Man., Canada. He is now with the Department of Electrical Engineering, McMaster University, Hamilton, Ont., Canada. certain approximations permitting one to identify the coefficients of the insertion loss function of an arbitrary inhomogeneous transformer with those of a suitable equal-ripple function. A system of nonlinear equations results, which leads to the unknown parameters of the transformer.

In this paper, the problem is formulated in very general terms for direct optimization by digital computer. The formulation is sufficiently flexible to facilitate the design of multisection, inhomogeneous, nonsynchronous, nonquarterwave rectangular waveguide transformers having nonideal junctions and possibly mismatched terminations. The only restriction is that any effect to be included be known and calculable. Constraints placed on the width, height, or length of any section need be dictated only by considerations for dominant mode propagation and the requirement of small (but not necessarily negligible) junction discontinuities. If reliable data is available for discontinuity effects due to simultaneous changes in width and height at a junction, it can be incorporated directly into the optimization process. This would obviate the usual need for subsequent correction using, for example, formulas due to Cohn [7].

Optimum equal-ripple solutions are presented to examples considered by Young [2], [4], Matthaei et al. [4], and Riblet [5]. The considerable improvements possible over their design procedures by the present more accurate but simpler formulation for computer optimization are thereby demonstrated. The optimization technique itself is a new direct search method called razor search, details of which are presented in a companion paper by Bandler and Macdonald [8]. Direct search methods, which do not require derivatives, have already found useful application to microwave network optimization involving complicated functions and constraints [9]. The problem here involves both noncommensurate and irrational transcendental functions of frequency. The objective function dictates that the maximum reflection coefficient of the transformer be minimized over a specified bandwidth. This maximum is located by an optimization strategy called ripple search [8]. The objective function is thus characterized by discontinuous partial derivatives [8]$[10]$. The razor search strategy was designed to handle such problems.

To conclude the introduction, a few words of explanation are in order about the meaning of "optimum" as used in this paper. All optimum designs presented here are constrained in one way or another as discussed in Section II. But Young's and Riblet's designs are also constrained. The essential difference lies in the fact that the present formulation has as its objective a minimax equal-ripple response over a predetermined frequency band satisfying the constraints selected for the particular problem. And in achieving this objective, no 
approximations are made concerning the behavior of the transformer at any given frequency other than those employed in predicting the final response, whether based on ideal or nonideal junctions. Thus, assuming proper convergence is obtained, effects such as the unexpected bandbroadening observed by Riblet [5] will not occur.

\section{General Formulation of the Problem}

\section{A. The Objective}

Fig. 1 represents the inhomogeneous rectangular waveguide transformer in terms of the geometrical parameters which can be varied. The objective is to find

$$
\check{U}=\min _{\boldsymbol{\phi}}(U)=\min _{\phi}\left\{\max _{\left[f l, f_{u}\right]}[|\rho(\boldsymbol{\phi}, f)|]\right\}
$$

where

$$
\phi=\left[a_{1}, b_{1}, l_{1}, a_{2}, b_{2}, l_{2}, \cdots a_{m}, b_{m}, l_{m}\right]^{T}
$$

where $a_{k}, b_{k}$ and $l_{k}$ are the width, height and length of the $k$ th waveguide section $(k=1,2, \cdots m), \rho$ is the reflection coefficient at the transformer input, $f$ is frequency, and $f_{l}$ and $f_{u}$ are the lower and upper band edges, respectively. Thus, as illustrated in Fig. 2, the objective is to obtain a set of parameter values $\breve{\phi}$ which minimizes $U$, which is the maximum magnitude of $\rho$ in the band.

\section{B. The Objective Function}

$U$ is obtained from the following considerations. Using the notation of Fig. 1

$$
\begin{array}{r}
Y_{I_{k}}=Y_{k} \frac{Y_{I_{k+1}}+j B_{k+1}+P_{k} Y_{k}}{Y_{k}+P_{k}\left(Y_{I_{k+1}}+j B_{k+1}\right)} \\
k=m, m-1, \cdots 1
\end{array}
$$

where

$P_{k}=j \tan \theta_{k}$

$Y_{f_{0}}$ is the characteristic admittance of the $k$ th section

$\theta_{k}$ is the electrical length of the $k$ th section

$Y_{I_{k}}$ is the input admittance to the $k$ th section

$B_{b}$ is the discontinuity susceptance at the $k$ th junction.

At the output terminals

$$
Y_{I_{m+1}}=y_{L} Y_{m+1}
$$

where $y_{L}$ is the complex normalized load admittance. At the input terminals we can define $\rho$ as

$$
\rho=\frac{y_{s}^{*} Y_{0}-\left(Y_{I_{1}}+j B_{1}\right)}{y_{s} Y_{0}+Y_{I_{1}}+j B_{1}}
$$

where $y_{s}$ is the complex normalized source admittance. Under matched conditions $y_{s}=1$ and $y_{L}=1$; otherwise the frequency dependencies of $y_{s}$ and $y_{L}$ must be specified. Under $H_{10}$-mode operation

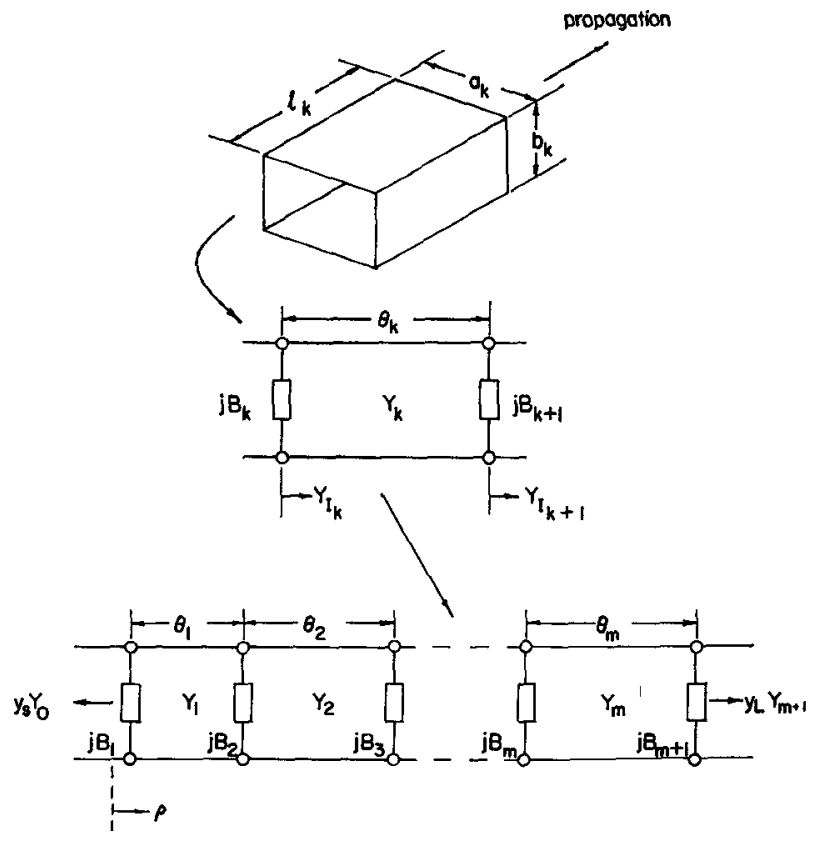

Fig. 1. Representation of the inhomogeneous rectangular waveguide impedance transformer.

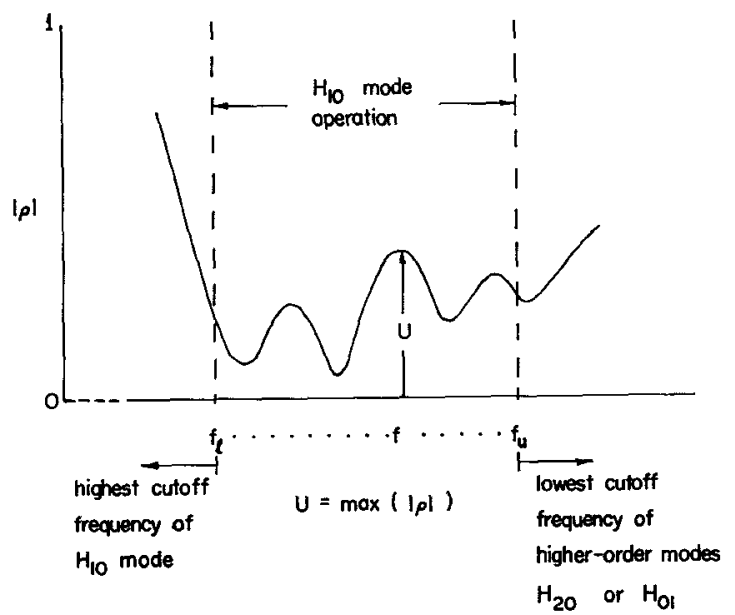

Fig. 2. The objective function $U$ to be minimized over some frequency band within the permissible operating range of the transformer.

$$
\begin{aligned}
Y_{k} & =Y\left(a_{k}, b_{k}, f\right) \\
\theta_{k} & =\theta\left(a_{k}, l_{k}, f\right) \\
B_{k} & =B\left(a_{\kappa}, b_{\kappa}, f\right) \quad \kappa=k-1, k .
\end{aligned}
$$

\section{The Constraints}

Constraints on the $a, b$ and $l$ dimensions must be such that

1) only $H_{10}$-mode propagation within the transformer band is allowed,

2) the network representation of the transformer as in Fig. 1 is valid,

3) sufficiently accurate descriptions of $Y, \theta$ and $B$ can be found.

1) Limits on Individual Parameters: Upper and lower limits on the geometrical parameters of the transformer are 


$$
\left.\begin{array}{rl}
\frac{\lambda_{l}}{2} & <a_{k}<\lambda_{u} \\
0 & <b_{k}<\frac{\lambda_{u}}{2} \\
0 & <l_{k}<\lambda_{u} / 2 \sqrt{1-\left(\lambda_{u} / 2 a_{k}\right)^{2}}
\end{array}\right\} k=1,2, \cdots m
$$

where $\lambda_{l}\left(=c / f_{l}\right)$ and $\lambda_{u}\left(=c / f_{u}\right)$ are the free space wavelengths at the lower and upper band edges, respectively. Constraints (10) and (11) ensure $H_{10}$-mode propagation and that the next higher order modes, namely the $H_{20}$-mode and the $H_{01}$-mode are cutoff, as shown in Fig. 2. Inequality (12) expresses the belief that optimum solutions will have the length of any section lying between zero and half the shortest guide wavelength in that section.

Given the input and output guide dimensions the chosen band $f_{l}$ to $f_{u}$ must, of course, conform to

$$
\left.\begin{array}{c}
\frac{\lambda_{l}}{2}<a_{k}<\lambda_{u} \\
0<b_{k}<\frac{\lambda_{u}}{2}
\end{array}\right\} \quad k=0, m+1 .
$$

2) Constraints for Small Symmetrical Steps: There still seems to be no data available for waveguide discontinuities involving simultaneous changes in the $a$ and $b$ dimensions. In order to use simple and reliable formulas for $Y$ and $\theta$, and in order to be able to superimpose individual values for discontinuity susceptances due to separate changes in the $a$ and $b$ dimensions at any junction with sufficient accuracy, the present discussion will restrict itself to small, but not necessarily negligible, symmetrical steps. To use the appropriate formulas from Marcuvitz [11] let

$$
\left.\begin{array}{l}
\frac{7}{10}<\frac{a_{k}}{a_{k-1}}<\frac{10}{7} \\
\frac{1}{2}<\frac{b_{k}}{b_{k-1}}<2
\end{array}\right\} k=1,2, \cdots m+1 .
$$

\section{The Functions $Y$ and $\theta$}

Riblet has shown [5] that the most suitable expression for the characteristic impedance $Z$ of a rectangular waveguide to fit in with the representation in Fig. 1 is $Z=b \lambda_{g}$, where $\lambda_{g}$ is the guide wavelength. This closely approximates a formula found in Marcuvitz [11, p. 296], and its validity was confirmed experimentally [12]. The result can also be confirmed from experimental results obtained by the author [13]. Thus we can write

$$
Y=\frac{1}{b \lambda_{0}}
$$

where

$$
\lambda_{g}=\frac{\lambda}{\sqrt{1-(\lambda / 2 a)^{2}}} .
$$

$H$-plane discontinuity effects (change in $a$-dimension) involve a shift in reference plane from the physical plane of the discontinuity [11, p. 296]. However, if (10), (13) and (15) are imposed, this shift becomes negligible for practical purposes. In this case

$$
\theta=\frac{2 \pi l}{\lambda_{g}} .
$$

The functional dependence of $Y_{k}$ and $\theta_{k}$ in (7) and (8) is now specified in (17) through (19).

The frequency dependent impedance ratio $R$ of the transformer is defined, using (17), as

$$
R=\frac{b_{m+1} \lambda_{g m+1}}{b_{0} \lambda_{g 0}}
$$

\section{E. The Discontinuity Susceptance B}

During optimization any of the nine situations depicted in Fig. 3 could arise. All possibilities must be converted to the form in Fig. 4 for the purpose of calculating the discontinuity susceptances from the formulas derived from Marcuvitz [11] and set out in the Appendix. For programming convenience, the correspondence of the variables of Fig. 3 with those of Fig. 4 is established in Table I.

Table I implies the following: the $H$-plane component $B_{h}$ of the discontinuity susceptance is calculated from (27) assuming the height of the wider waveguide to be constant across the junction, and the $E$-plane component $B_{e}$ is calculated from (28) assuming the width of the higher waveguide to be constant across the junction. Note that there are no conflicting choices to be made when a junction has a discontinuity of one kind only.

We have, therefore, at the $k$ th junction the approximate susceptance

$$
B_{k}=B_{h k}+B_{e k}
$$

where $B_{h k}$ and $B_{e k}$ are calculated from (27) and (28) using the appropriate variables obtained from Table I.

\section{F. Further Considerations}

Both Young [2] and Riblet [5] have observed that we have more degrees of freedom than are strictly required to find "optimum" transformer designs. One thing that can be done is to hold the values of $a$ constant such that the $H$-plane discontinuities are reduced. Thus, one would optimize only with the $b$ and $l$ parameters with

$$
\frac{\lambda_{l}}{2}<a_{k}=c_{k}<\lambda_{\iota} \quad k=1,2, \cdots m
$$

where the $c_{k}$ are constant.

Another possibility is to specify that

$$
0<\sum_{k=1}^{m} l_{k} \leq L<\sum_{k=1}^{m} \lambda_{u} / 2 \sqrt{1-\left(\lambda_{u} / 2 a_{k}\right)^{2}}
$$

where $L$ is some maximum allowable overall length. Alternatively one might specify that 

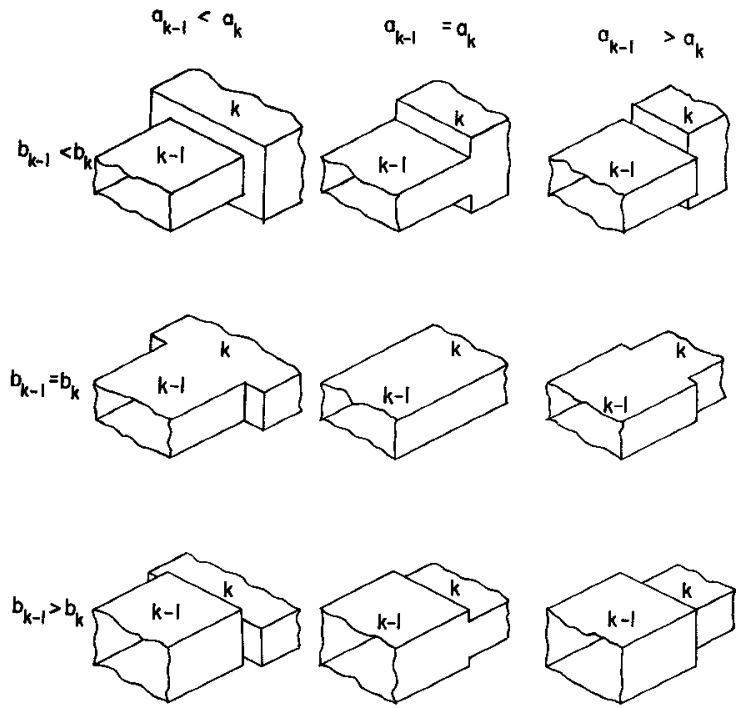

Fig. 3. Types of waveguide junction allowed during optimization.
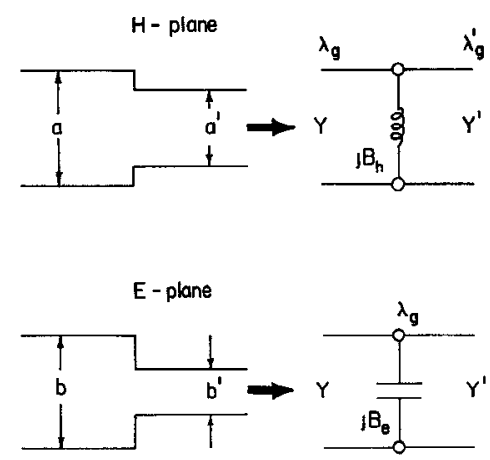

Fig. 4. Representation of $H$-plane and $E$-plane junctions.

TABLE I

Correspondence of the Variables of Fig. 3 With Those of Fig. 4 for the Purpose of Estrmating the Discontinutty Susceptances UsING (27) AND (28)

\begin{tabular}{|c|c|c|c|c|}
\hline$H$-Plane & $a$ & $a^{\prime}$ & $\lambda_{g}$ & $Y$ \\
\hline $\begin{array}{l}a_{k-1}<a_{k} \\
a_{k-1}=a_{k}\end{array}$ & $a_{b}$ & \multicolumn{3}{|c|}{ no $H$-plane discontinuity } \\
\hline$a_{k-1}>a_{k}$ & $a_{k-1}$ & $a_{k}$ & $\lambda_{g k-1}$ & $Y_{k-1}$ \\
\hline E-Plane & $b$ & $b^{\prime}$ & $\lambda_{g}$ & $Y$ \\
\hline $\begin{array}{l}b_{k-1}<b_{k} \\
b_{k-1}=b_{k}\end{array}$ & & $\begin{array}{c}b_{k-1} \\
\text { no } E \text {-plan }\end{array}$ & $\begin{array}{l}\lambda_{g k} \\
\text { continuity }\end{array}$ & $Y_{k}$ \\
\hline$b_{k-1}>b_{k}$ & $b_{k-1}$ & $b_{k}$ & $\lambda_{g k-1}$ & $Y_{k-1}$ \\
\hline
\end{tabular}

$0<l_{k} \leq l_{u k}<\lambda_{u} / 2 \sqrt{1-\left(\lambda_{u} / 2 a_{k}\right)^{2}} \quad k=1,2, \cdots m$

where $l_{u k}$ is an upper limit on $l_{k}$ such that

$$
\sum_{k=1}^{m} l_{u k}=L
$$

Such constraints can lead to nonsynchronous transformers.

Under certain circumstances, some of the constraints need not be imposed during the optimization. It is unlikely, for example, that the section lengths will violate (12) if not constrained assuming the initial values are sensibly chosen to lie in the region of $\lambda_{g k} / 4$ at some frequency between $f_{l}$ and $f_{u}$. It may be found unnecessary to monitor (15) and (16) continuously, particularly if the $a_{k}$ are fixed as in (22). These decisions could be made on the basis of the known behavior of equal length homogeneous transformers.

If the incorporation of constrained variables into the computer program becomes necessary, the following possibilities exist: the constrained variables may be transformed into new variables which are not constrained and in which the optimization is carried out [9], [14], but perhaps the simplest way is to impose a penalty on the objective function when any constraint is violated, e.g.,

$$
U=1
$$

which represents the worst possible transformer design.

\section{G. The Computer Program}

The program for optimizing inhomogeneous rectangular waveguide impedance transformers was written following the theory outlined in this section. Additional details concerning the structuring of the program are found in the Appendix.

\section{Optimized Design EXAMPLeS}

\section{A. Young's Two-Section Transformers [2]}

The predicted VSWR versus frequency for both of Young's two-section designs are reproduced in Fig. 5. The designs resulted from modifications to his exact maximally flat solutions using the homogeneous quarter-wave transformer tables as a guide. In Young's first design (Fig. 5(a)) both sections are quarter-wave at $1.3 \mathrm{GHz}$. In his second (Fig. 5(b)) both sections are quarter-wave at $8.5 \mathrm{GHz}$. His design procedure takes $Z$ as $b \lambda_{g} / a \lambda$, therefore, his predicted responses in Fig. 5 use this definition of $Z$. All junctions are assumed ideal.

Table II shows the dimensions of the transformers. Note that the broad dimensions were fixed at Young's values (see (22)) and that the starting values for the four varied parameters are Young's design values. ${ }^{1}$ Using $Z=b \lambda_{g}$, the first transformer was optimized over the band 1.255 to 1.365 $\mathrm{GHz}$, and the second from 8.16 to $9.25 \mathrm{GHz} .^{2}$ The optimization process was assumed to have converged when all the reflection coefficient minima became less than 0.001 , corresponding to a VSWR of about 1.002. ${ }^{3}$ For the first transformer, a maximum VSWR of 1.0230 was obtained, for the second a maximum VSWR of 1.0470 . The optimum responses and the transformer impedance ratios are plotted in Fig. 5.

As Table II shows, a slight decrease in overall length was obtained in Example 1, indicating that the substantial im-

${ }^{1}$ The fact that junction 2 violates (15) was ignored for this example.

${ }^{2}$ Young did not specify any desired frequency band in advance, therefore, the author selected the equal-ripple band in Example 1, and the band predicting a VSWR of 1.09 in Example 2.

${ }^{3}$ For all practical purposes, therefore, we have two zeros of reflec- 


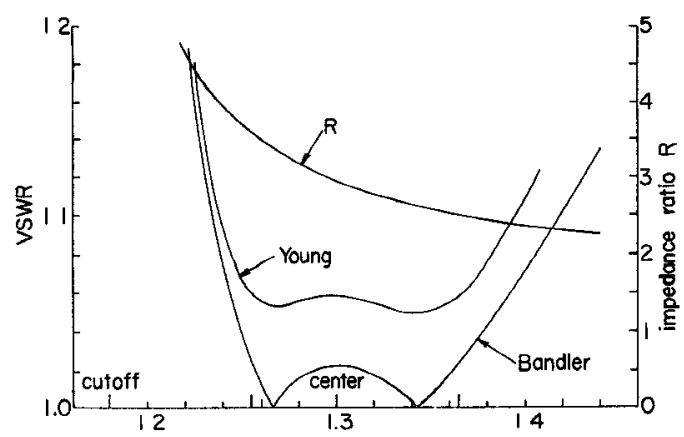

(a)

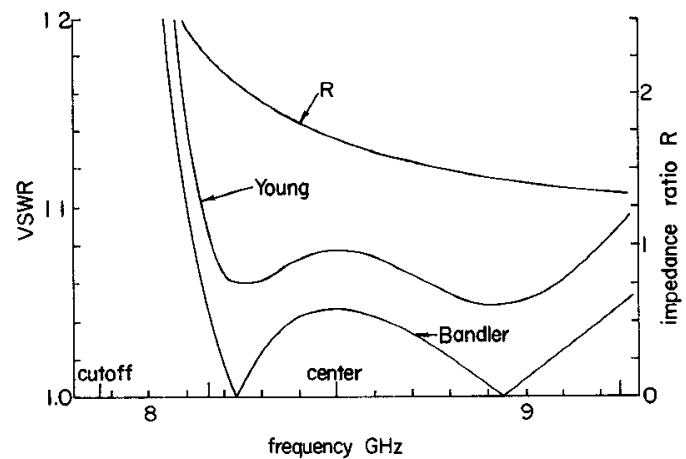

(b)

Fig. 5. Responses of Young's two-section transformers (based on $\left.Z=b \lambda_{\theta} / a \lambda\right)$ and comparison with those of the author's optimized designs (Table II).

TABLE II

The Dimensions in Centimeters of Young's Two-Section TRANSFormers BEFore AND AFTER OPTIMIZATION

\begin{tabular}{|c|c|c|c|c|c|c|}
\hline \multirow{2}{*}{$\begin{array}{l}\text { Ex- } \\
\text { ample }\end{array}$} & \multirow[b]{2}{*}{$k$} & & \multicolumn{2}{|c|}{$b_{k}$} & \multicolumn{2}{|c|}{$I_{k}$} \\
\hline & & $a_{k}$ & Start & Finish & Start & Finish \\
\hline \multirow{4}{*}{1} & 0 & 20.32 & \multicolumn{2}{|c|}{5.080} & \multicolumn{2}{|c|}{$\infty$} \\
\hline & 1 & 20.32 & 8.920 & 7.43255 & 7.025 & 6.90951 \\
\hline & 2 & 13.5661 & 8.04418 & 8.14517 & 11.03 & 11.0770 \\
\hline & 3 & 12.70 & & & & \\
\hline \multirow{4}{*}{2} & 0 & 2.286 & \multicolumn{2}{|c|}{1.016} & \multicolumn{2}{|c|}{$\infty$} \\
\hline & 1 & 2.159 & 1.110 & 1.08486 & 1.5331 & 1.46572 \\
\hline & 2 & 1.95834 & 1.03886 & 1.04832 & 2.0387 & 2.14850 \\
\hline & 3 & 1.905 & \multicolumn{2}{|c|}{1.016} & \multicolumn{2}{|c|}{$\infty$} \\
\hline
\end{tabular}

provement in response is not attributable to any allowance for increase in length. ${ }^{4}$

\section{B. Riblet's Three-Section Transformer [5]}

The predicted response of Riblet's three-section transformer is reproduced in Fig. 6 together with his design specification of a VSWR of 1.03 over $500 \mathrm{MHz}$ centered at $6.175 \mathrm{GHz}$. It was designed assuming ideal junctions, then corrected for junction discontinuities by Cohn's method [7]. The author took the dimensions of Riblet's corrected design and calculated the response including discontinuity effects according the rules established in Section II. The response is so close to the ideal one that it is not shown separately in

${ }^{4}$ It is also interesting to note that the maximum height of the transformer $b_{2}$ is less than the maximum height before optimization $b_{1}$.

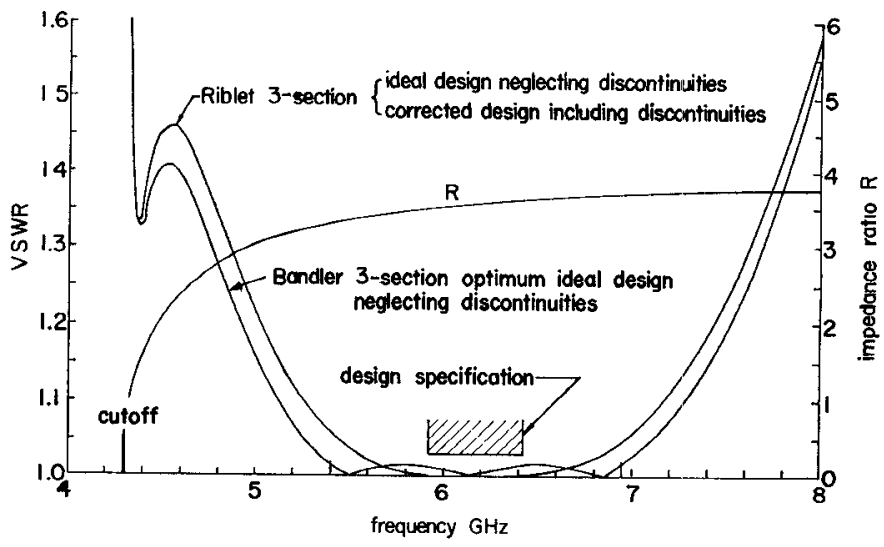

Fig. 6. The response of Riblet's three-section design. The ideal design neglecting discontinuities exhibits for all practical purposes the same response as the corrected design including discontinuities. The response of an optimumideal design neglecting discontinuities (Table III) is also shown.

Fig. 6. Riblet's measured response [5] is also very close to the predicted ones. Thus the validity of both Riblet's and the author's approximation concerning the superposition of small symmetrical discontinuities is verified, at any rate, for this example.

It may be observed in Fig. 6 that Riblet has unexpectedly exceeded his design specification by a substantial margin. The question arises: can his specification be met by a shorter design? First, however, as an example, Riblet's design was optimized keeping the waveguide widths fixed for convenience and assuming ideal junctions. The outcome of optimizing over the band 5.4 to $6.95 \mathrm{GHz}$ is shown in Fig. 6. A maximum VSWR of 1.0157 was obtained, and the minima are less than 1.0015. The relevant dimensions are given in Table III. The improvement in bandwidth is almost 45 percent for a mere one percent increase in overall length. ${ }^{5}$

An interesting phenomenon first pointed out to the author by Young [15] is the behavior of the response near cutoff. The explanation is probably the following: the transformer impedance ratio $R$ is very close to 1 at the minimum nearest the cutoff frequency. Here the guide wavelengths are fairly high causing the transformer to be electrically short. The result is a more nearly matched system which could give rise to the dip in VSWR.

One and two-section designs were investigated assuming ideal junctions. The results are plotted in Fig. 7. First, a single section transformer having a width equal to that of the output waveguide was optimized from 5.925 to $6.425 \mathrm{GHz}$. As shown in Fig. 7(a) it fails to meet the specification. In addition, its first junction violates (16). Second, a two-section transformer having its first section width equal to the input waveguide width and its second section width equal to the output waveguide width was tried over the required band. Its optimum response is shown in Fig. 7(b). With a maximum VSWR of 1.0070 it looks attractive. However, its second junction violates (16). Third, a two-section transformer hav-

${ }^{5}$ By raising the lower band edge very slightly, the overall length could be decreased to Riblet's value without significantly changing the percentage bandwidth improvement. 
TABLE III

The Dimensions in Centimeters of Optimized Two- and Three-Section Transformers Satisfying Riblet's Specification

\begin{tabular}{|c|c|c|c|c|c|c|}
\hline \multirow{3}{*}{$\begin{array}{l}\text { Input guide } \\
\text { Three-section } \\
\text { Ideal }\end{array}$} & \multirow{2}{*}{$\begin{array}{c}k \\
0 \\
\end{array}$} & \multirow{2}{*}{$\frac{a_{k}}{.48488}$} & \multicolumn{2}{|c|}{$\operatorname{tart}^{b_{k}}$ Finish } & \multicolumn{2}{|c|}{ Start $^{l_{k}}$ Finish } \\
\hline & & & \multicolumn{2}{|c|}{0.508} & \multicolumn{2}{|c|}{$\infty$} \\
\hline & $\begin{array}{l}1 \\
2 \\
3\end{array}$ & $\begin{array}{l}3.51536 \\
3.65 \\
3.90144\end{array}$ & $\begin{array}{l}0.59182 \\
0.96266 \\
1.64846\end{array}$ & $\begin{array}{l}0.598878 \\
0.956654 \\
1.62020\end{array}$ & $\begin{array}{l}1.67906 \\
1.6262 \\
1.55055\end{array}$ & $\begin{array}{l}1.69602 \\
1.63686 \\
1.55684\end{array}$ \\
\hline $\begin{array}{l}\text { Two-section } \\
\text { Ideal }\end{array}$ & $\begin{array}{l}1 \\
2\end{array}$ & $\begin{array}{l}3.6 \\
3.8\end{array}$ & $\begin{array}{l}0.75 \\
1.25\end{array}$ & $\begin{array}{l}0.711967 \\
1.39486\end{array}$ & $\begin{array}{l}1.6 \\
1.6\end{array}$ & $\begin{array}{l}1.65733 \\
1.59002\end{array}$ \\
\hline $\begin{array}{l}\text { Two-section } \\
\text { Nonideal }\end{array}$ & $\begin{array}{l}1 \\
2\end{array}$ & $\begin{array}{l}3.6 \\
3.8\end{array}$ & $\begin{array}{l}0.711967 \\
1.39486\end{array}$ & $\begin{array}{l}0.713153 \\
1.39661\end{array}$ & $\begin{array}{l}1.65733 \\
1.59002\end{array}$ & $\begin{array}{l}1.56044 \\
1.51621\end{array}$ \\
\hline Output guide & 4,3 & 4.0386 & \multicolumn{2}{|c|}{2.0193} & \multicolumn{2}{|c|}{$\infty$} \\
\hline
\end{tabular}

(a)

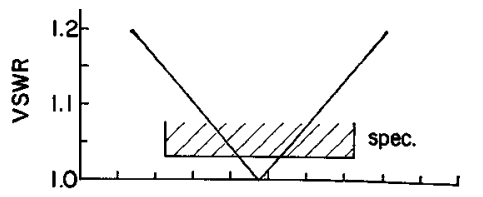

(b)

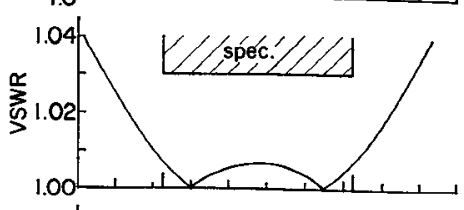

(c)

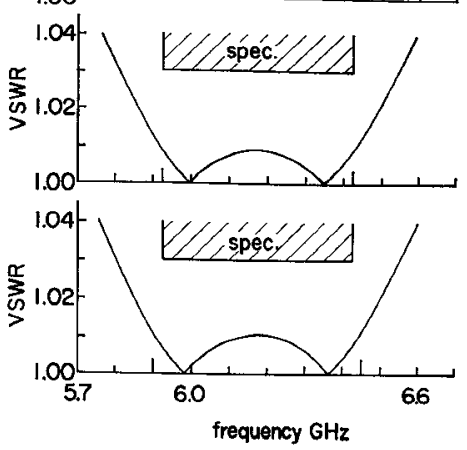

Fig. 7. Responses of some optimized transformers assuming ideal junctions attempting to satisfy Riblet's specification, (a) one-section with $a_{1}=a_{2}$, (b) two-section with $a_{1}=a_{0}$ and $a_{2}=a_{8}$, (c) and (d) twosection with $a_{1}=3.6 \mathrm{~cm}$ and $a_{2}=3.8 \mathrm{~cm}$. (See text for further details.)

ing $a_{1}=3.6 \mathrm{~cm}$ and $a_{2}=3.8 \mathrm{~cm}$ was optimized. Its response is shown in Fig. 7(c). The maximum VSWR is 1.0089 and it satisfies (16). It also meets the specification required by Riblet. [Fig. 7(d) shows another possible design, optimized over 5.9 to $6.45 \mathrm{GHz}$ using broad dimensions as for Fig. 7(c)].

The dimensions of the two-section transformer are given in Table III. The response of this ideal design when junction discontinuities are included is shown in Fig. 8. It violates the specification. Therefore, it was re-optimized with discontinuity effects accounted for in the manner of Section II, using the dimensions of the ideal design as a starting point. ${ }^{6}$ The outcome is plotted in Fig. 8, and the corresponding dimensions are found in Table III. The predicted response of the nonideal design including discontinuities is so close to

\footnotetext{
'Note that changes in height as well as length were allowed, unlike the common practice of correcting only the lengths.
}

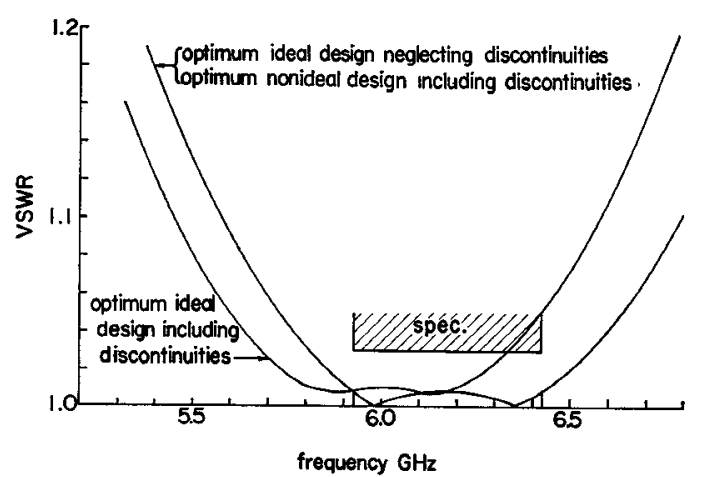

Fig. 8. Responses of optimized two-section transformers satisfying Riblet's specification. The corresponding dimensions are given in Table III.

the ideal design neglecting discontinuities that only one curve is shown. In fact, the maximum VSWR is 1.0090 in the former case as compared with about 1.0089 in the latter.

Note that for all intents and purposes the response minima for Figs. 7 and 8 are zeros of reflection.

\section{The Five-Section Transformer in Matthaei et al. [4, p.} 333-334]

This five-section transformer consists of an optimum three-section homogeneous transformer followed by a twosection inhomogeneous transformer. The transformer was designed assuming ideal junctions using $Z=b \lambda_{g} / a \lambda$ and having a nominal center frequency of $6.15 \mathrm{GHz}$. Its response is shown in Fig. 9. It exhibits a VSWR of less than 1.05 over a 20 percent band.

It was decided to try optimizing a three-section transformer over the range 5.7 to $7.2 \mathrm{GHz}$, which exceeds the band of the five-section design. The widths of the sections were fixed, for convenience, so that the step changes were equal from one section to the next. The dimensions of the resulting transformer are shown in Table IV, and the corresponding response in Fig. 9. The maximum pass band VSWR is 1.0340 . All-round improvements in desired bandwidth, VSWR and overall length have been obtained.

It is interesting to observe that none of the minima in the response are zeros of reflection. However, it should be recalled that the objective is to minimize the maximum re- 


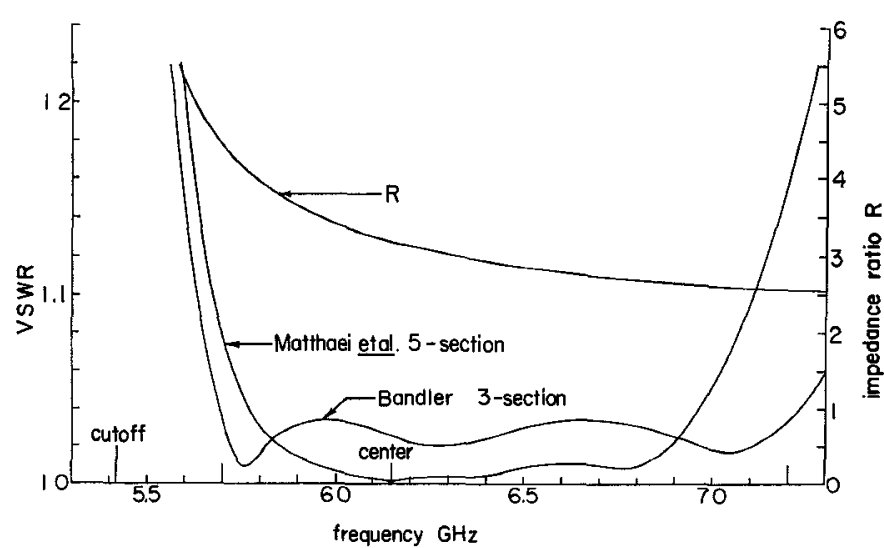

Fig. 9. Comparison of the response of a five-section transformer (based on $Z=b \lambda_{g} / a \lambda$ ) taken from Matthaei et al. with that of a three-section transformer (Table IV), designed by the author. Both designs assume ideal junctions.

TABLE IV

The Dimensions in Centimeters of the Optimized Three-Section Transformer Whose Response is Shown in Fig. 9

\begin{tabular}{c|c|c|c}
\hline \hline$k$ & $a_{k}$ & \multicolumn{1}{|c|}{$b_{k}$} & $l_{k}$ \\
\hline 0 & 3.48488 & 0.762 & $\infty$ \\
1 & 3.30581 & 0903176 & 1.54879 \\
2 & 3.12674 & 1.37093 & 1.58375 \\
3 & 2.94767 & 1.73609 & 1.64590 \\
4 & 2.76860 & 1.60325 & $\infty$ \\
\hline
\end{tabular}

flection coefficient, without consideration for the minima. Exhaustive searches in the vicinity of the apparent optimum were conducted to see (with the widths constant at their original values) whether a better response having lower minima was possible. The results are negative. ${ }^{7}$

\section{Young's Seven-Section Homogeneous Transformer [16]}

This example is considered not so much to demonstrate significant improvement due to computer optimization (since exact analytic methods are available for homogeneous transformer design) but to test the author's computer program including discontinuity effects.

The seven-section transformer was designed by a firstorder method resulting in the continuous curve in Fig. 10. A computer program to evaluate the response including discontinuity effects was not available to Young at that time. So he estimated it by computing the response of an ideal transformer obtained by adding the length corrections the "wrong" way. The author calculated this response and compared it with the dashed curve in Fig. 10, which is the response of the ideal design including discontinuities. For all practical purposes the responses are the same and are, therefore, not shown separately.

The curves of Fig. 11 are probably of more interest. One is the response of Young's corrected design including discontinuity effects. Although the ripples are within the nominal VSWR of 1.01, there is a slight deterioration at the edges. Using Young's final design parameter values as a starting point, the optimized response shown in Fig. 11 was arrived

${ }^{7}$ It is quite likely that if the section widths were also allowed to vary, zeros could have been obtained, together with a "better" optimum.

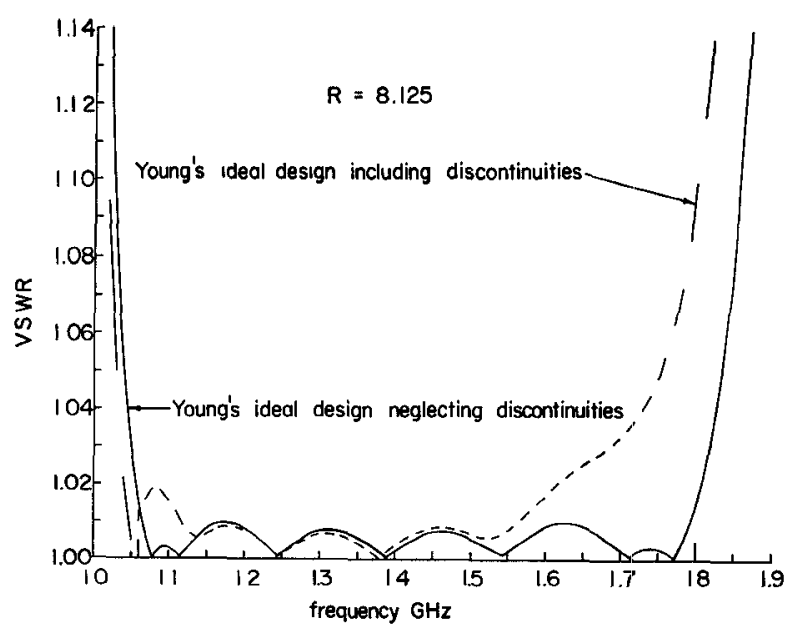

Fig. 10. The response of a seven-section ideal homogeneous transformer designed by Young, with and without consideration for junction discontinuity effects.
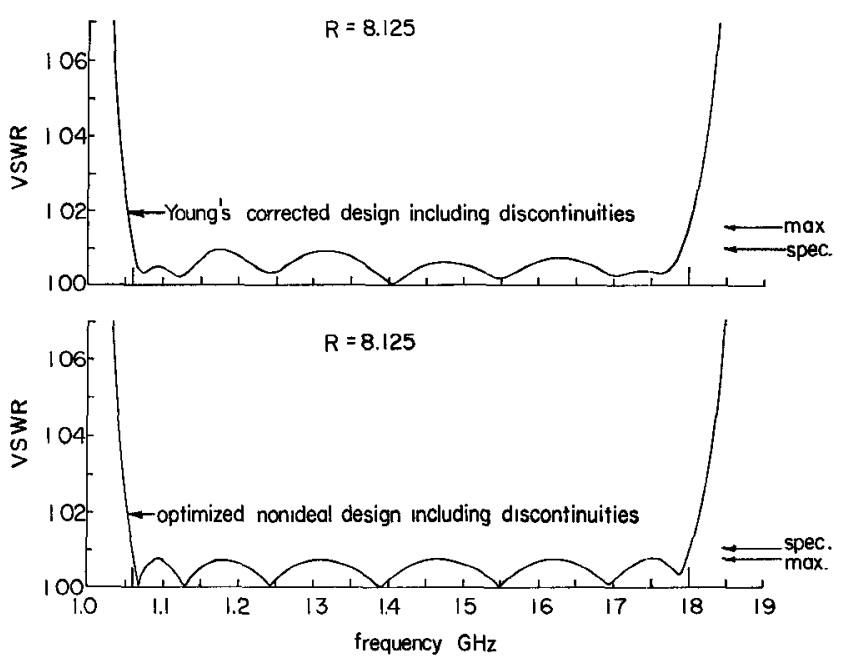

Fig. 11. The response of Young's corrected seven-section transformer including discontinuity effects. Also shown is the response obtained by the author after optimization including discontinuity effects. The dimensions of both designs are given in Table V.

at. ${ }^{8}$ Discontinuity effects were included in the optimization, and both the $b$ and $l$ parameters were allowed to vary. Table $\mathrm{V}$ compares the dimensions for both designs.

The maximum pass band VSWR of the author's design is 1.0078. The failure of the minimum at the high frequency end to be substantially a zero of reflection is thought to be due to the inclusion of discontinuity effects which, as seen from Fig. 10, are more severe at higher frequencies. The peaks are all within 0.0002 of 1.0078 .

\section{E. Computer Execution Times}

With the exception of the seven-section example just described all the VSWR maxima agreed to five significant figures, rather more than required for practical purposes. ${ }^{9}$

${ }^{8} \mathrm{An}$ inhomogeneous design is not presented because (10) would have had to be violated.

${ }^{9}$ Rounding off the optimized dimensions to six significant figures as shown in the tables may cause the five figure agreement to deteriorate very slightly in some cases. In view of the approximation made, for example with regard to discontinuity effects, such accuracy could probably not be justified experimentally anyway. 
TABLE V

The Dimensions in Centimeters of the Seven-Section Homogeneous TRANSFORMERS $(a=16.51 \mathrm{~cm})$ WhOSE Responses ARE SHOWN IN Fig. 11

\begin{tabular}{l|l|l|l|l}
\hline \hline \multirow{2}{*}{$k$} & \multicolumn{2}{|c|}{ Young } & \multicolumn{2}{c}{ Bandler } \\
\cline { 2 - 5 } & \multicolumn{1}{|c|}{$b_{k}$} & \multicolumn{1}{c}{$l_{k}$} & $b_{k}$ & $l_{k}$ \\
\hline 0 & 8.255 & $\infty$ & 8.255 & $\infty$ \\
1 & 7.85876 & 7.16534 & 7.85315 & 7.20134 \\
2 & 6.60400 & 7.07898 & 6.58323 & 7.05331 \\
3 & 4.66598 & 6.91134 & 4.65041 & 6.89954 \\
4 & 2.89560 & 6.92404 & 2.89463 & 6.91110 \\
5 & 1.79832 & 7.00532 & 1.80056 & 7.00313 \\
6 & 1.27000 & 7.06374 & 1.27067 & 7.06581 \\
7 & 1.06680 & 7.10692 & 1.06691 & 7.12327 \\
8 & 1.016 & $\infty$ & 1.016 & $\infty$ \\
\hline
\end{tabular}

Running times on the IBM $360 / 65$ were $^{10}$ : for two-section, four-variable transformers in the region of $\frac{1}{2}$ minute; for three-section, six-variable transformers in the region of three minutes. The times are strongly dependent on the choice of an initial design and on the scaling of the parameters. Some initial effort spent in working out an approximate design using, for example, the homogeneous transformer theory is recommended. Good scaling can decrease the computing times by as much as two-thirds. For three figure agreement between the maxima there will be further reduction in computing time. ${ }^{11}$

\section{CONCLUSIONS}

This paper has concerned itself with the computer optimization of inhomogeneous waveguide transformers. A general formulation of the problem has been presented which should enable the design of such transformers to meet most practical specifications. A systematic method for including discontinuity effects directly into the optimization process has been described. Several design examples taken from the literature have been considered and discussed. Note that the author's results are not necessarily the "best possible" optima, since in the examples presented the waveguide widths were fixed in advance. Even if all available parameters had been allowed to vary there could still be some doubt if the constraints of Section II are imposed. An experimental transformer was not constructed as sufficient experimental evidence of the accurate predictability of the response of inhomogeneous waveguide transformers [1], [5] and waveguide junctions [12], [13] exists for the kind of dimensions considered in this paper. Through the use of constraints both realizable and practical transformers are always guaranteed.

The approach used in this paper should also find application in the computer-aided design of broadband microwave matching or equalizing networks consisting of noncommensurate components and for which exact synthesis techniques may be unavailable.

\footnotetext{
${ }^{10}$ The programs were run using FORTRAN IV-G under full OS-MFT. Running times could probably be reduced by a third by using FORTRAN IV-H.

${ }^{11}$ Depending on the constraints imposed in a particular problem it may not be possible to equalize the maxima even to three figures.
}

\section{APPENDIX}

\section{Computation of Discontinuity Susceptances}

The formula used for computing the discontinuity susceptance due to small symmetrical $H$-plane steps is derived from Marcuvitz [11, p. 296] as

$$
\begin{aligned}
b_{h}= & \frac{B_{h}}{Y} \approx-\frac{\lambda_{g}}{2 a} \frac{\beta^{2}(1+\beta) \ln \frac{2}{\beta}}{1-\frac{\beta}{2}} \\
& \cdot\left(1-\frac{27}{8} \cdot \frac{Q+Q^{\prime}}{1+8 \ln \frac{2}{\beta}}\right) ; \quad \beta \ll 1
\end{aligned}
$$

where

$$
\begin{aligned}
& \beta=1-\frac{a^{\prime}}{a} \\
& Q=1-\sqrt{1-\left(\frac{2 a}{3 \lambda}\right)^{2}} \\
& Q^{\prime}=1-\sqrt{1-\left(\frac{2 a^{\prime}}{3 \lambda}\right)^{2}}
\end{aligned}
$$

and where $a, a^{\prime}, Y$ and $\lambda_{g}$ are as shown in Fig. 4(a), and $Y$ and $\lambda_{g}$ are defined by (17) and (18), respectively. The range of application of (27) is maintained by (10), (11), (13), (14) and (15).

The formula for computing the discontinuity susceptance due to small symmetrical $E$-plane steps is obtained from Marcuvitz [11, p. 308] as

$$
b_{c}=\frac{B_{e}}{Y} \approx \frac{2 b}{\lambda_{\theta}}\left(\frac{\delta}{2}\right)^{2}\left[\frac{2 \ln \frac{2}{\delta}}{1-\delta}+1+\frac{17}{16}\left(\frac{b}{\lambda_{g}}\right)^{2}\right] ;
$$

where $\delta=1-b^{\prime} / b$ and where $b, b^{\prime}, \lambda_{g}$ and $Y$ are as shown in Fig. 4(b). The range of application of (28) is maintained by (10), (11), (13), (14), and (16).

\section{Description of the Computer Program}

In addition to the package of subroutines comprising the razor search strategy and the ripple search strategy described by Bandler and Macdonald [8], the following programs were required:

1) The Main Program: This reads all the data which defines the problem, and the constants and scale factors for the optimization process. A logical variable is set true if junction discontinuities are to be considered, and false otherwise. The decision as to which parameters are to be allowed to vary is made in this program prior to calling RAZOR. This program also instructs the printing out of the results.

2) A Function Subprogram for Calculating $|\rho(f)|$ : Equations (3)-(6) and (17)-(20) appear in this function subpro- 
gram. $|\rho(f)|$ is the objective function which is called by the Ripple Search package. The logical variable of 1) controls the inclusion of discontinuity effects by calling or not calling 3).

3) A Function Subprogram for Calculating $B_{k}$ : This function subprogram implements the rules laid out in Table I for orienting the various junction configurations that are depicted in Fig. 3. Having called 4) or 5) as appropriate, $B_{k}$ is calculated as in (21).

4) A Function Subprogram for Calculating $b_{h}$ : The normalized discontinuity susceptance due to small symmetrical $H$-plane steps is calculated here by (27).

5) A Function Subprogram for Calculating $b_{e}$ : The normalized discontinuity susceptance due to small symmetrical $E$-plane steps is calculated here by (28).

6) A Logical Function Subprogram for Testing Constraints: The constraints in (10)-(16) which are most likely to be violated are tested here, and on the basis of any violation a design can be rejected or a penalty can be imposed on the objective function during optimization.

The program was written in FORTRAN IV for use on the IBM 360/65. Further details on how these programs tie up with the razor search and ripple search programs need not be given here since they are essentially the same as for the homogeneous transmission-line transformer examples discussed by Bandler and Macdonald [8]. The reader may also wish to refer to the description of a program [18] which calculates the response of an inhomogeneous cascade of rectangular waveguides with or without junction discontinuity effects in accordance with the theory presented in this paper.

\section{ACKNOWLEDGMENT}

The author wishes to express his appreciation to those colleagues at the University of Manitoba who have shown their interest in this work. In particular, the author has benefited from stimulating discussions with Dr. A. Wexler on waveguide problems and numerical methods; with Prof. E. Bridges on waveguide problems; and with P. A. Macdonald, who also contributed expert programming assistance, on optimization techniques. The author must also acknowledge Dr. R. E. Collin [17] who suggested the idea of optimizing inhomogeneous waveguide transformers by computer. Fur- ther thanks are extended to Dr. H. J. Riblet [12] who made available his results on waveguide impedance measurements, and to Dr. L. Young [15] for his helpful comments. The cooperation of the Institute for Computer Studies of the University of Manitoba is acknowledged.

\section{REFERENCES}

[1] L. Young, "Optimum quarter-wave transformers," IRE Trans. Microwave Theory and Techniques, vol. MTT-8, pp. 478-483, September 1960.

[2] —-, "Inhomogeneous quarter-wave transformers of two sections," IRE Trans. Microwave Theory and Techniques, vol.MTT-8, pp. 645-649, November 1960.

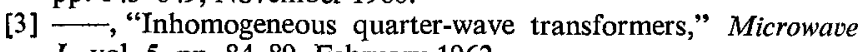
J., vol. 5, pp. 84-89, February 1962.

[4] G. L. Matthaei, L. Young, and E. M. T. Jones, Microwave Filters, Impedance Matching Networks, and Coupling Structures. New York: McGraw-Hill, 1964, ch. 6.

[5] H. J. Riblet, "A general design procedure for quarter-wavelength inhomogeneous impedance transformers having approximately equal-ripple performance," IEEE Trans. Microwave Theory and Techniques, vol. MTT-13, pp. 622-629, September 1965.

[6] L. Young, "Stepped impedance transformers and filter prototypes," IRE Trans. Microwave Theory and Techniques, vol. MTT10, pp. 339-359, September 1962.

[7] S. B. Cohn, "Optimum design of stepped transmission-line transformers," IRE Trans. Microwave Theory and Techniques, vol. MTT-3, pp. 16-21, April 1955.

[8] J. W. Bandler and P. A. Macdonald, "Optimization of microwave networks by razor search," this issue, pp. 552-562.

[9] J. W. Bandler, "Optimization methods for computer-aided design," this issue, pp. 533-552.

[10] J. W. Bandler and P. A. Macdonald, "Cascaded noncommensurate transmission-line networks as optimization problems," IEEE Transactions on Circuit Theory (Correspondence), vol. CT-16, pp. 391-394, August 1969.

[11] N. Marcuvitz, Waveguide Handbook, M.I.T. Rad. Lab. Ser., vol. 10. New York: McGraw-Hill, 1951, pp. 296-298, 307-308.

[12] H. J. Riblet, Microwave Development Labs. Inc., Needham Heights, Mass., private communication.

[13] J. W. Bandler, "Abrupt transitions in rectangular waveguides," Electrical Engineering Dept., Imperial College, London, England, 3rd year Project Rept., June 1963.

[14] - "Optimum noncommensurate stepped transmission-line transformers," Electronics Letters, vol. 4, pp. 212-213, May 1968.

[15] L. Young, Stanford Research Institute, Menlo Park, Calif., private communication.

[16] - "Practical design of a wide-band quarter-wave transformer in waveguide," Microwave J., vol. 6, pp. 76-79, October 1963.

[17] R. E. Collin, Case Western Reserve University, Cleveland, Ohio, private communication.

[18] J. W. Bandler and P. A. Macdonald, "Response program for an inhomogeneous cascade of rectangular waveguides," this issue, pp. 646-649. 